\title{
Constraints on environs around SN 2011fe and SN 2014J from radio modeling and observations
}

\author{
Esha Kundu ${ }^{1}$, Peter Lundqvist ${ }^{1}$ and Miguel A. Pérez-Torres ${ }^{2,3}$ \\ ${ }^{1}$ Department of Astronomy and The Oskar Klein Centre, AlbaNova, Stockholm University, \\ SE-10691 Stockholm, Sweden \\ email: esha.kundu@astro.su.se, peter@astro.su.se \\ ${ }^{2}$ Instituto de Astrofísica de Andalucía, Glorieta de las Astronomía, s/n, E-18008 Granada, \\ Spain \\ ${ }^{3}$ Visiting Scientist: Departamento de Física Teorica, Facultad de Ciencias, Universidad de \\ Zaragoza, Spain \\ email: torres@iaa.es
}

\begin{abstract}
The radio non-detection of two Type Ia supernovae (SNe) SN 2011fe and SN 2014J has been modeled considering synchrotron radiation from shock accelerated electrons in the SN shock fronts. With $10 \%$ each of the bulk kinetic energy in electric and magnetic fields, a very low density of the medium around both the SNe has been estimated from the null detection of radio emission, around 1 and 4 years after the explosion of SNe 2014J and 2011fe, respectively. Keeping the fraction of energy in electrons fixed at $10 \%$, a medium with particle density $\sim 1$ $\mathrm{cm}^{-3}$ is found when $1 \%$ of the post shock energy is in magnetic fields. In case of a wind medium, the former predicts the mass loss rate $\dot{M}$ to be $<10^{-9} \mathrm{M}_{\odot} \mathrm{yr}^{-1}$, and the latter gives an upper limit $\sim 10^{-9} \mathrm{M}_{\odot} \mathrm{yr}^{-1}$, for wind velocity of $100 \mathrm{~km} \mathrm{~s}^{-1}$, for both the SNe. The tenuous media obtained from this study favor the double degenerate as well as a spin up/down model for both SNe 2011fe and 2014J.
\end{abstract}

Keywords. stars: circumstellar matter, supernovae: general, supernovae: individual (SN 2011fe, SN 2014J)

\section{Introduction}

Type Ia supernovae (SNe) are accepted to be the thermonuclear explosion of white dwarfs (WDs) composed of carbon and oxygen (Hoyle \& Fowler 1960). They are widely used as standard candles in cosmology (Riess et al. 1998, Perlmutter et al. 1999), and are one of the main sources of metals in the universe. Hence Type Ia SNe play an important role in terms of chemical evolution of galaxies. As it is understood so far, the successful disruption of a WD requires a companion star. In the single degenerate (SD) scenario the companion is a non-degenrate star, i.e., a main sequence or a asymptotic giant branch star, from which the WD accretes matter before explosion. Classically, the WD explodes when it reaches the Chandrashekar mass limit. Close to this limit, the central density is high enough for carbon to ignite, which obliterates completely the WD (Whelan \& Iben 1973). A second possible formation channel consists of another WD as a companion, with the total mass of the WDs exceeding the Chandrasekhar mass. In this case, known as the double degenerate (DD) scenario, the two WDs spiral each other and emit gravitational waves. This causes the reduction of angular momentum from the system which reduces the orbital separation. Eventually, the two WDs merge and this results in a successful thermonuclear runaway (Iben \& Tutukov 1984, Webbink 1984). The most likely type of 
progenitor of SNe Ia is not yet clear, which restrains their usage as cosmological standard candles.

The two formation channels are expected to yield significantly different circumbinary medium characteristics. Namely, in the SD scenario a fraction of the companion mass is usually lost to the surrounding medium via winds or Roche-lobe overflow. This results in a high density circumbinary medium. On the contrary, the merger model predicts a very clean, low-density environment around the progenitor star. The interaction of high velocity $\mathrm{SN}$ ejecta with this medium causes a strong shock wave to move through the circumstellar medium (CSM), which then sweeps the material up into a thin shell. The radio and X-ray emission from this shocked region thus allow to measure the density of the ambient medium, from which one could eventually distinguish the SNe Ia formation channels.

While radio emission has been detected from many core-collapse supernovae (e.g., SN 1993J (Bartel et al. 1994)), SN 2011dh (Soderberg et al. 2012, Krauss et al. 2012)), no SN Ia has ever been detected at these wavelengths (Panagia et al. 2006, Chomiuk et al. 2016). Since the expected emitted radiation is weak, the large distance to most known SNe Ia makes it challenging to detect them. In this regard the two nearest young SNe Ia, SNe 2011fe and 2014J at distances of 6.4 (Shappee \& Stanek 2011) and 3.4 Mpc (Marion et al. 2015), respectively, provide a unique opportunity to study these events in detail.

The non-detection of early-time radio and X-ray emission from SN 2011fe constrains the mass loss rate $(\dot{M})$ of the SN progenitor to be less than $6 \times 10^{-10} \mathrm{M}_{\odot} \mathrm{yr}^{-1}$ (Chomiuk et al. 2012) and $2 \times 10^{-9} \mathrm{M}_{\odot} \mathrm{yr}^{-1}$ (Margutti et al. 2012), respectively, considering a wind velocity $\left(v_{w}\right)$ of $100 \mathrm{~km} \mathrm{~s}^{-1}$. For a merger scenario they obtained a medium with density, $n_{\mathrm{ISM}}$, less than 6 and $150 \mathrm{~cm}^{-3}$, respectively. Applying a similar formalism for the radio emission from SN 2014J, Pérez-Torres et al. 2014 estimated $\dot{M}<7 \times$ $10^{-10}\left(v_{w} / 100 \mathrm{~km} \mathrm{~s}^{-1}\right)^{-1} \mathrm{M}_{\odot} \mathrm{yr}^{-1}$ and $n_{\mathrm{ISM}}<1.3 \mathrm{~cm}^{-3}$. Although the low density medium predicted from early observations favours a merger scenario over a SD channel for both $\mathrm{SNe}$, the radio models contain a few poorly constrained shock parameters, the most relevant being the magnetic field energy density. In this situation, observations at late epochs ( $t \gtrsim 1 \mathrm{yr}$ after the explosion), in addition to the early time observations, are important in constraining the environment and hence the progenitor scenario.

\section{Ejecta and CSM structure}

The structure of the inner ejecta can be parametrized in different ways, but rather than assuming a power-law (Pérez-Torres et al. 2014) or an exponential density structure (Chomiuk et al. 2016), we have taken the density structure from explosion models, namely from N100 (Röpke et al. 2012, Seitenzahl et al. 2013) and a violent merger model (Pakmor et al. 2012). These two test the SD and DD formation channels of Type Ia, respectively. The outer ejecta profile is assumed to follow a power law distribution, i.e., $\rho_{e j} \propto r^{-n}$, where $n$ is the power law index and $r$ is the radius of the SN. In our study $n$ can vary in the range 12 - 14. In this paper, we refer the N100 + power law structure for outer ejecta the N100 model and the violent merger + power law profile the merger model.

As discussed in $\S 1$, in case of a SD channel, the winds from the progenitor system could result in a high density circumbinary medium. Therefore, for a constant value of $\dot{M}$ and $v_{w}$ the density of the CSM could be written as $\rho(r)=\dot{M} /\left(4 \pi r^{2} v_{w}\right)$. For the DD channel the density of the ambient medium is constant and is characteristic of the interstellar medium (ISM). This implies that in case of DD scenario $\rho(r)=n_{\mathrm{ISM}} \mu$, where $\mu$ represents the mean atomic weight of the circumbinary medium. Therefore, for both 
media we can write $\rho(r)=A r^{-s}$, where $s=2$ (0) represents the wind (constant density) medium.

\section{Radio modeling}

Shocks are ideal places where particles can be accelerated efficiently. The interaction between SN ejecta and CSM launches a strong shock, called forward shock, into the ambient medium. The pressure behind this shock drives another shock, known as reverse shock, back into the ejecta. As a result a shocked shell is formed. This shell contains the shocked outer part of the ejecta and shocked circumstellar matter. When ejecta with a power law density structure interact with an ambient medium, having a power law profile for the density, the evolution of this shell can be described by a self-similar structure (Chevalier 1982). This implies that the shock radius evolves as $r_{s} \propto A^{1 /(s-n)} t^{(n-3) /(n-s)}$, where $t$ represents time since explosion.

We modeled the radio emission assuming synchrotron radiation from shock accelerated relativistic electrons. All electrons in the post shock region are considered to be accelerated and follow a power law distribution in energy, i.e., $d N / d E=N_{0} E^{-p}$, where $N_{0}$ and $E$ represent the normalization constant of the distribution and the electron energy, respectively. $p$ is the power law index. For a non-relativistic shock, having a compression factor of $\eta=4$, diffusive shock acceleration (DSA) predicts $p=2$. However, as discussed in Ellison \& Reynolds (1991), the presence of a sub-shock in a cosmic ray dominated shock could result in a particle spectrum, for low energy particles, with an index steeper than 2. Moreover, in case of Type $\mathrm{Ib} / \mathrm{c}$ SNe it is found from the observations that $p=3$ (Chevalier \& Fransson 2006). We therefore assume $p=3$ for our study.

The radio luminosity, with synchrotron self absorption (SSA) as the dominant absorption mechanism, from an optically thin shell having an outer radius $r_{s}$ is given by

$$
L_{\nu, \text { thin }}=\frac{8 \pi^{2} k T_{\mathrm{bright}} \vartheta_{\nu} r_{s}^{2}}{c^{2} f\left(\frac{\nu_{\text {peak }}}{\nu_{\mathrm{abs}}}\right)} \nu_{\mathrm{abs}, 0}^{(p+3) / 2} \nu^{-(p-1) / 2},
$$

with

$$
\nu_{\mathrm{abs}, 0}=\left(2 \Delta r \varkappa(p) N_{0} B^{(p+2) / 2}\right)^{2 /(p+4)}
$$

(Pérez-Torres et al. 2014), where $T_{\text {bright }}$ and $\vartheta_{\nu}$ being the brightness temperature and the ratio between the radio intensity from a given part of the shell to that from the centre part, respectively. $\Delta r$ is the thickness of the radio emitting shell and it is $\propto r_{s} . \varkappa(p)$ and $B$ represent the SSA coefficient and magnetic field strength, respectively. $k$ is the Boltzmann constant and $c$ represents the speed of light in vacuum. For a wind medium $(s=2)$, and with $p=3$, the optically thin radio luminosity, eqn. 3.1 , can be written as

$$
L_{\nu, \text { thin }} \propto\left(\frac{n-3}{n-2}\right)^{3.86} T_{\text {bright }} \epsilon_{\text {rel }}^{1.71} \epsilon_{\mathrm{B}}^{1.07}\left(\dot{M} / v_{w}\right)^{\frac{1.93 n-8.43}{n-2}} t^{-\frac{(n+2.57)}{n-2}} \nu^{-1},
$$

and for a constant density medium $(s=0)$

$$
L_{\nu, \text { thin }} \propto\left(\frac{n-3}{n}\right)^{3.86} T_{\text {bright }} \epsilon_{\text {rel }}^{1.71} \epsilon_{\mathrm{B}}^{1.07}\left(n_{\mathrm{ISM}}\right)^{\frac{1.93 n-8.43}{n}} t^{\frac{(2.86 n-25.3)}{n}} \nu^{-1}
$$

(Kundu et al. 2017), where $\epsilon_{\mathrm{rel}}$ and $\epsilon_{\mathrm{B}}$ being the fraction of bulk kinetic energy in relativistic electrons and magnetic fields, respectively. If $u_{t h}$ represents the post shock thermal energy density, then $\epsilon_{\mathrm{B}}=u_{B} / u_{t h}=B^{2} /\left(8 \pi u_{t h}\right)$ and the fraction of post shock 


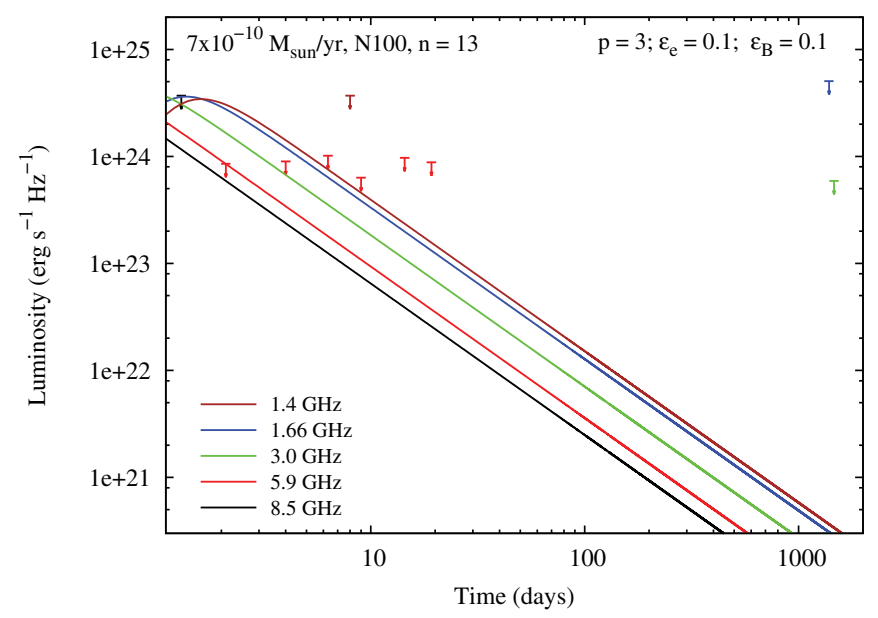

Figure 1. Light curves for SN 2011fe, in solid lines, predicted by the N100 model, for $n=13$ and $\epsilon_{\mathrm{e}}=\epsilon_{\mathrm{B}}=0.1$, when the SN plows through a wind medium. The upper limits are from Horesh et al. (2012), Chomiuk et al. (2012) and Kundu et al. (2017).

energy that has been channeled into electrons is $\epsilon_{\mathrm{e}}=\epsilon_{\mathrm{nrel}}+\epsilon_{\mathrm{rel}}=u_{e} / u_{t h}$, where $u_{B}$ and $u_{e}$ are magnetic and electric field energy densities, respectively. Here, $\epsilon_{\mathrm{nrel}}$ is the fraction of post shock energy in non-relativistic electrons (non-relativistic electrons are those with kinetic energy $E_{k}<m_{e} c^{2}$ (三 $E_{\text {rest }}$ ), where $m_{e}$ is the mass of the electron). Just after the explosion the shock velocity is very high, which often implies $\epsilon_{\mathrm{e}}=\epsilon_{\mathrm{rel}}$. With time, as the shock gets decelerated, a part of the electron population becomes non-relativistic. This suggests $\epsilon_{\mathrm{rel}}=\epsilon_{\mathrm{e}}\left(\frac{E_{m i n}}{E_{\mathrm{rest}}}\right)^{(p-2)}$ (Kundu et al. 2017), where $E_{\text {min }}$ is the minimum energy of the electron population. Recent hybrid simulations have shown that in a non-relativistic parallel/quasi-parallel shock (i.e., the shock normal and the magnetic field vector are almost aligned) a maximum of $10-20 \%$ of the post shock energy can be converted into accelerated ions and the acceleration efficiency becomes negligible in a perpendicular shock (Caprioli \& Spitkovsky 2014). Considering these facts we assume that for electrons the acceleration efficiency is $10 \%$, i.e., $\epsilon_{\mathrm{e}}=0.1$. For magnetic fields we consider two cases: (i) $\epsilon_{\mathrm{B}}=0.1$ and (ii) $\epsilon_{\mathrm{B}}=0.01$. This means that in one case it is assumed that the post-shock energy has been equally shared between electric and magnetic fields, i.e., $\epsilon_{\mathrm{e}}=\epsilon_{\mathrm{B}}=0.1$ and for the second situation the energy in the magnetic fields is considered to be less than that in electric fields, i.e., $\epsilon_{\mathrm{e}}=0.1$ and $\epsilon_{\mathrm{B}}=0.01$.

Fig. 1 represents the radio light curves predicted by the N100 model for SN 2011fe, along with observational upper limits. As visible in the figure, the upper limit in luminosity measured around 2 days after the explosion at $5.9 \mathrm{GHz}$ constrains the maximum mass loss rate of the system. This has been also noted by Chomiuk et al. (2012) and Pérez-Torres et al. (2014). With $n=13$ and $\epsilon_{\mathrm{e}}=\epsilon_{\mathrm{B}}=0.1$ it is found that $\dot{M}<7 \times 10^{-10}$ $\mathrm{M}_{\odot} \mathrm{yr}^{-1}$ for $v_{w}=100 \mathrm{~km} \mathrm{~s}^{-1}$ (Kundu et al. 2017). Considering $n=12$ and keeping other parameters fixed, the light curves from the merger model are shown in fig. 2. In this case, the radio upper limit at $3 \mathrm{GHz}$, obtained around 4 years after the explosion, limits $n_{\mathrm{ISM}}$ to be less than $0.23 \mathrm{~cm}^{-3}$ (Kundu et al. 2017). For our study we assume a constant value of $T_{\text {bright }}=5 \times 10^{10} \mathrm{~K}$.

For SN 2014J the upper limits on $\dot{M}$ and $n_{\mathrm{ISM}}$ are derived from the observations done around 8 and 410 days after the explosion of the SN (Pérez-Torres et al. 2014, Kundu et al. 2017). 


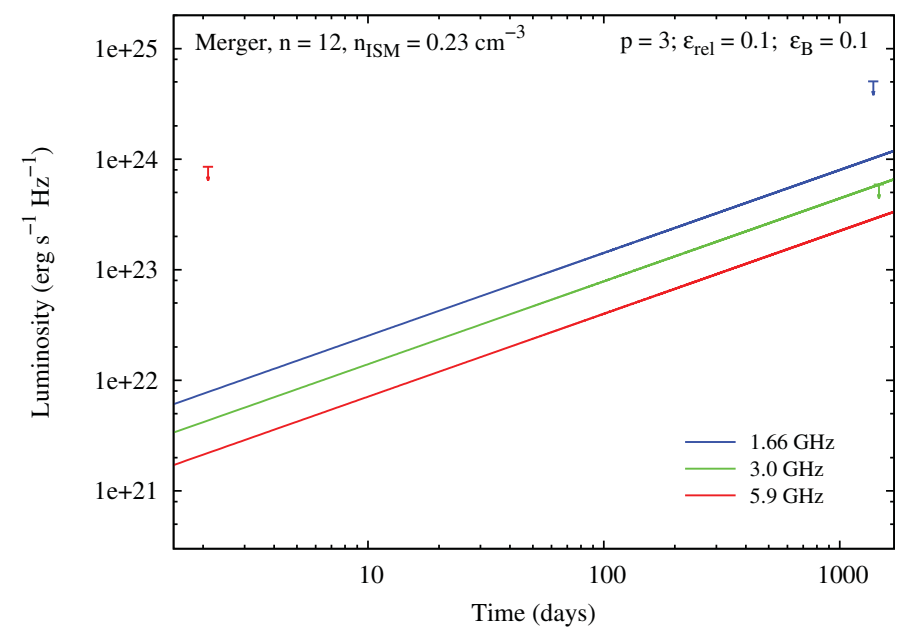

Figure 2. Light curve from a merger model for SN 2011fe when the SN expands in a constant density medium. The upper limits are from Chomiuk et al. (2012) and Kundu et al. (2017). (Only a few upper limits on radio luminosity are plotted here as these are the most constraining data points.)

\section{Implications}

It has been found from the radio modeling that the circumbinary media around both SNe 2011fe and 2014J are tenuous. The upper limit on mass loss rate of both SNe inferred from our modeling is $10^{-9}\left(v_{w} / 100 \mathrm{~km} \mathrm{~s}^{-1}\right)^{-1} \mathrm{M}_{\odot} \mathrm{yr}^{-1}$ when $\epsilon_{\mathrm{B}}=0.1$ and $n$ varies in the range $12-14$ (Kundu et al. 2017). For $\epsilon_{\mathrm{B}}=0.01$ the upper limit on $\dot{M}$ is $\sim 10^{-9}$ for $v_{w}=100 \mathrm{~km} \mathrm{~s}^{-1}$. In case of a constant density medium, the upper limit on $n_{\mathrm{ISM}}$, around both SNe, are estimated to be $\sim 0.35 \mathrm{~cm}^{-3}$ and $\sim 1 \mathrm{~cm}^{-3}$ when $\epsilon_{\mathrm{B}}=0.1$ and 0.01, respectively (Kundu et al. 2017). This implies that both SNe 2011fe and 2014J could be a result of mergers of two WDs, which predicts a very low density medium around the explosion site. On the other hand, the tenuous medium can also be due to recurrent novae. In this case, a cavity may exist close to the progenitor star. However, from optical nebular spectra this is found to be a less likely progenitor system for both SNe 2011fe and 2014J (Lundqvist et al. 2015). Another possibility within the SD scenario is the spin up/down model (Justham 2011). In this case, the WD spins down by losing angular momentum, and for some cases the spin down time could be more than $10^{9}$ yrs (Ilkov \& Soker 2012). During this time the CSM may diffuse into the ISM. Therefore, the surroundings could attain a density of the order of ISM density. However, if we assume $n_{\mathrm{ISM}}$ to be $\sim 1 \mathrm{~cm}^{-3}$ (as indicated from other evidences in Kundu et al. (2017)), the radio non-detection at late time could point toward a low magnification efficiency of magnetic fields in SN shocks.

\section{References}

Bartel, N., Bietenholz, M. F., Rupen, M. P., Conway, J. E., et al. 1994, Nature, 369, 584

Caprioli, D. \& Spitkovsky, A. 2014, ApJ, 783, 91

Chevalier, R. A. \& Fransson C. 2006, ApJ, 651, 381

Chevalier, R. A. 1982, ApJ, 258, 790

Chomiuk, L., Soderberg, A. M., Moe, M., et al. 2012, ApJ, 750, 164

Chomiuk, L., Soderberg, A. M., Chevalier, R. A., et al. 2015, ApJ, 821, 119

Ellison, D. C. \& Reynolds, S. P. 1991, ApJ, 382, 242 
Horesh, A., Kulkarni, S. R., Fox, D. B., et al. 2012, apj, 746, 21

Hoyle, F. \& Fowler W. A. 1960, ApJ, 132, 565

Iben, I., Jr. \& Tutukov, A. V. 1984, ApJS, 54, 335

Ilkov, M. \& Soker, N. 2011, MNRAS, 419, 1695

Justham, S. 2011, ApJ, 730, L34

Krauss, M. I., Soderberg, A. M., Chomiuk, l., et al. 2012, ApJ, 750, L40

Kundu, E., Lundqvist, P., Pérez-Torres, M. A., \& Herrero-Illana, R., \& Alberdi A. 2017, arXiv:1705.04204

Lundqvist, P., Nyholm, A., Taddia, F., et al. 2015, A\&A, 577, 39

Margutti, R., Soderberg, A. M., Chomiuk, L., et al. 2012, ApJ, 751, 134

Margutti, R., Parrent, J., Kamble, A., et al. 2014, ApJ, 790, 52

Marion, G. H., Sand, D. J., Hsiao, E. Y., et al. 2015, ApJ, 798, 39

Pakmor, R., Kromer, M., Taubenberger, S., Sim, S. A., Röpke, F. K., \& Hillebrandt, W. 2012, ApJ (Letters), 747, L10

Panagia, N., Van Dyk, S. D., Weiler, K. W., et al. 2006, ApJ, 646, 369

Perlmutter, S., Aldering, G., Goldhaber, G., et al. 1999, ApJ, 517, 565

Pérez-Torres, M. A., Lundqvist, P., Beswick, R. J., et al. 2014, ApJ, 792, 38

Riess, A., Filippenko, A. V., Challis, P., et al. 1998, AJ, 116, 1009

Röpke F. K., Kromer, M., Seitenzahl, I. R., Pakmor, R., et al. 2012, ApJ (Letters), 750, L19

Seitenzahl, I. R., Ciaraldi-Schoolmann, F., Röpke, F. K., et al. 2013, MNRAS, 429, 1156

Shappee, B. J. \& Stanek, K. Z. 2011, ApJ, 733, 124

Soderberg, A. M., Margutti, R., Zauderer, B. A., et al. 2012, ApJ, 752, 78

Webbink, R. F. 1984, ApJ, 277, 355

Whelan, J. \& Iben, I., Jr. 1973, ApJ, 186, 1007 